\title{
Influences of Donor and Recipient Gene Polymorphisms on Tacrolimus Dosing and Pharmacokinetics in Asian Liver Transplant Patients
}

\author{
Mei-Ling Yee ${ }^{1 \#}$, Hui-Hui Tan ${ }^{2}$, Wan-Jin Sia ${ }^{1}$, Wai-Ping Yau ${ }^{3}$ \\ ${ }^{1}$ Department of Pharmacy, Singapore General Hospital, Singapore city, Singapore \\ ${ }^{2}$ Department of Gastroenterology and Hepatology, Singapore General Hospital, Singapore city, Singapore \\ ${ }^{3}$ Department of Pharmacy, Faculty of Science, National University of Singapore, Singapore city, Singapore \\ Email: \#yee.mei.ling@sgh.com.sg
}

Received June 1, 2013; revised July 2, 2013; accepted July 10, 2013

Copyright (C) 2013 Mei-Ling Yee et al. This is an open access article distributed under the Creative Commons Attribution License, which permits unrestricted use, distribution, and reproduction in any medium, provided the original work is properly cited.

\begin{abstract}
Background: Tacrolimus pharmacokinetics has large inter-individual variability. Objectives: This study aimed to investigate the impact of donor and recipient gene polymorphisms on tacrolimus dosing and pharmacokinetics in Asian liver transplant patients. Methods: Steady-statetacrolimus concentrations at 0, 1, 2, 4 and $6 \mathrm{~h}$ were measured. Pharmacokinetic parameters were estimated with a one-compartment linear model using WinNonlin 6.1 program. DNA from donor liver and recipient blood samples were genotyped for CYP3A and ABCB1 polymorphisms. Results: A total of 13 donors and 17 recipients were included. Donor genotypes influenced tacrolimus trough concentration-to-dose (C0/D) ratio only at Week 1 . Patients with liver grafts from CYP3A5 expressors $\left({ }^{*} 1^{*} 1\right.$ and $\left.{ }^{*} 1{ }^{*} 3\right)$ achieved lower mean C0/D ratio than those with grafts from non-expressors ( $\left.{ }^{*} 3^{*} 3\right)(64.48$ versus $129.21(\mathrm{mcg} / \mathrm{L}) /(\mathrm{mg} / \mathrm{kg} / \mathrm{day}), \mathrm{P}=0.040)$. Hence, the dose required to achieve target concentration in patients with donor genotype CYP3A5 expressors was higher than non-expressors $(0.12$ versus $0.08 \mathrm{mg} / \mathrm{kg}, \mathrm{P}=0.045)$. Recipient with ABCB1-C3435T genotype TT demonstrated higher apparent oral clearance of tacrolimus as compared to genotype CC $(17.7$ versus $7.9 \mathrm{~L} / \mathrm{h}, \mathrm{P}=0.033)$. Conclusions: Donor liver CYP3A polymorphism could potentially affect tacrolimus C0/D ratio as early as the first week post liver transplant. Genotyping of liver donors may be useful to achieve the optimal drug concentration during this critical period.
\end{abstract}

Keywords: Tacrolimus; Gene Polymorphisms; Liver Transplant; Pharmacokinetics

\section{Introduction}

Over the years, tacrolimus has become well established as the primary immunosuppressant employed by most liver transplant centers. It was proven to be efficacious with better graft and patient survival rates post liver transplantation [1-3]. However, optimal dosing with therapeutic monitoring in the first few days post-liver transplant, which is of vital importance, becomes especially challenging due to its large intra- and inter-individual variability in pharmacokinetic profile and narrow therapeutic index.

In recent years, studies were conducted to identify factors that lead to tacrolimus pharmacokinetic variability.

"Corresponding author.

*Gene Polymorphisms on Tacrolimus Dosing \& Pharmacokinetics.
Tacrolimus is a substrate of both cytochrome P450 3A (CYP3A) enzyme and ATP-binding cassette sub-family $\mathrm{B}$ member 1 (ABCB1) transporter. Advances in pharmacogenetics discovered several single nucleotidepolymorphisms in CYP3A subfamily and ABCB1. Studies have shown that the gene polymorphisms of CYP3A5 and $\mathrm{ABCB} 1$ contribute to the variability of tacrolimus dose-adjusted concentrations $[4,5,8,9]$. Most of the studies on the influences of gene polymorphisms were conducted in the immediate post transplant period, ranging from Week 1 to Week 5 [4-8], while some others have not specified time post liver transplant $[9,10]$. After transplantation, the liver graft recovers over a course of several days. The donor T-cell chimerism following liver transplantation is very common within the first 3 weeks. The metabolic capacity of liver graft may change over time post-transplant. It would be of clinical interest to 
understand changes in pharmacokinetic parameters in relation to gene polymorphisms in an Asian population. Although some studies have been conducted in China, Japan and Korean liver transplant populations [4-6,9], as well as Singapore and Korean renal transplant populations [8-9], the influence of both donor and recipient gene polymorphisms on the pharmacokinetics of tacrolimus remains to be clearly defined.

The aim of this study is to investigate the impact of gene polymorphisms on tacrolimus dosing and pharmacokinetics in Asian liver transplant patients.

\section{Materials and Methods}

\subsection{Patients}

This is a single-center cohort study with patients recruited from a tertiary referral center for liver transplant. All liver transplant recipients were started on tacrolimus within the first week of transplant and were stable on oral tacrolimus for at least 3 months at the time of enrolment.

Patients were included only if they were 3 months post-transplant and had been taking the same dose of tacrolimus for more than 7 days up to the time of enrolment to ensure steady-state condition. Patients were excluded if 1) they were non-Asians; 2) they received multi-organ transplant; 3) there was evidence of graft rejection (acute cellular, ductopenia or chronic) within 3 months prior to study participation; 4) they had active infection, autoimmune hepatitis or active viral/metabolic hepatitis up to 1 month before study; 5) they had proven surgical complications (biliary, arterial or venous with corresponding radiological evidence); 6) they had renal impairment with estimated creatinine clearance $<30 \mathrm{~mL} / \mathrm{min}$ as assessed by the Cockcroft-Gault Equation for more than 1 month prior to study participation; 7) they had significant alcohol intake ( $>20 \mathrm{~g} /$ day or $>140 \mathrm{~g} /$ week); 8) they were on medicationsthat are documented tohave significant clinical interactions with tacrolimus (i.e. alter tacrolimus level by at least $50 \%$ from baseline) for more than 1 month prior to study participation; or 9) they were on alternative/complementary medicine (e.g. herbal, traditional, health supplements and homeopathy) that were not part of standard medical care. All patients recruited provided written informed consent. The study was approved by the institute Centralised Institutional Review Board.

\subsection{Pharmacokinetic Study Design and Analysis}

On the day of pharmacokinetic investigation, venous blood samples $(3 \mathrm{~mL})$ were collected into ethylenediaminetetraacetic acid-containing Vacutainer tubes at 0 (pre-dose), 1, 2, 4 and $6 \mathrm{~h}$ after administration of the morning dose of tacrolimus on an empty stomach, i.e. 1 hour before or 2 hours after meal. For patients receiving different morning and evening doses of tacrolimus, an additional blood sample at $12 \mathrm{~h}$ after the morning dose was collected. Whole blood concentrations of tacrolimus at the targeted time-points $(\mathrm{Ct})$, i.e. $\mathrm{C} 0, \mathrm{C} 1, \mathrm{C} 2, \mathrm{C} 4, \mathrm{C} 6$ and $\mathrm{C} 12$, respectively, were measured by microparticle enzyme immunoassay using IMx analyzer (Abbott Laboratories, Abbott Park, IL) at Biochemistry Laboratory of institute. Measurement made with immunoassay was reported to be highly correlated with measurements made with high-performance liquid chromatographic mass spectrometry method (correlation coefficient: 0.987 ) [15]. For patients whose morning and evening doses were the same, $\mathrm{C} 12$ was assumed to be the same as $\mathrm{C} 0$ as the trough concentrations were reported to be not significantly different between the morning and evening doses of tacrolimus in liver transplant patients [16].

\subsection{Pharmacokinetic Model}

Pharmacokinetic parameters were estimated with a onecompartment, first-order elimination pharmacokinetic model using the Phoenix WinNonlin program (Version 6.1, Pharsight Corporation, CA).

\subsection{Genotyping}

DNA was extracted from donor livers and recipient blood samples using QIAampDNA Blood Kit (Qiagen, Germany). Polymerase chain reaction restriction fragment length polymorphism analysis was applied to determine genotypes. The CYP3A5 $5^{*} 3$ (A6986G in intron 3) and ABCB1 exon 12 (C1236T), exon 21 (G2677T/A) and exon 26 (C3435T) variant alleles were identified using an allelic discrimination assay.

\subsection{Statistical Analysis}

Statistical analyses were carried out using the SPSS software (version 18, SPSSInc, IL). Patient demographic and biochemical data were summarized using descriptive statistics with continuous variables reported as mean ( \pm $\mathrm{SD}$ ) and categorical variables as percentages. Tacrolimus $\mathrm{C} 0$ and weight-adjusted dose (D) at Week 1, Week 2, Month 1, Month 2 and more than 3 months post transplant were collected. Comparisons of $\mathrm{C} 0$-to-body weightadjusted dose $(\mathrm{C} 0 / \mathrm{D})$ ratio between 2 genotype groups, among 3 genotype groups and combination of donor/ recipient genotype groups were analysed with MannWhitney test and Kruskal-Wallis test, respectively. P < 0.05 was considered to be statistically significant.

\section{Results}

\subsection{Patient Characteristics}

From April 2010 to February 2011, 55 patients were followed up at the institutional liver transplant service. A total of 21 patients were excluded for: not being on tac- 
rolimus $(\mathrm{n}=10)$, being on tacrolimus for less than 3 months $(\mathrm{n}=4)$, significant drug interactions (fluconazole and voriconazole, $(n=3)$, hepatitis $C$ infection $(n=1)$, portal vein thrombosis $(n=1)$, renal dysfunction on dialysis $(n=1)$ or multi-organ transplant $(n=1)$. Out of 34 eligible patients, 17 patients who provided written con- sent were included in the study. As donor liver tissue samples were obtained retrospectively, only a total of 13 donor liver tissue samples were available at the time of study. Patient demographic and biochemical data are presented in Table 1. Majority of the patients was Chinese male $(n=12,70.6 \%)$ and received cadaveric liver

Table 1. Demographic and biochemical data of study transplant recipients $(n=17)$.

\begin{tabular}{|c|c|c|c|}
\hline & $\mathrm{n}$ & $\%$ & Mean \pm SD \\
\hline Age (years) & & & $53 \pm 9$ \\
\hline \multicolumn{4}{|l|}{ Gender } \\
\hline Male & 15 & 88.2 & \\
\hline Female & 2 & 11.8 & \\
\hline \multicolumn{4}{|l|}{ Race } \\
\hline Chinese & 14 & 82.4 & \\
\hline Malay & 1 & 5.9 & \\
\hline Indian & 1 & 5.9 & \\
\hline Korean & 1 & 5.9 & \\
\hline \multicolumn{4}{|l|}{ Transplant type } \\
\hline Cadaveric & 14 & 82.4 & \\
\hline Living-donor & 2 & 11.8 & \\
\hline Split graft & 1 & 5.9 & \\
\hline \multicolumn{4}{|l|}{ Indication for transplant } \\
\hline Hepatitis B with hepatocellular carcinoma & 7 & 41.2 & \\
\hline Hepatitis B & 3 & 17.6 & \\
\hline Hepatitis B/alcoholic liver disease & 2 & 11.8 & \\
\hline Primary biliary cirrhosis (PBC) & 2 & 11.8 & \\
\hline Hepatitis C & 1 & 5.9 & \\
\hline PBC/autoimmune hepatitis & 1 & 5.9 & \\
\hline Refractory ascites in cryptogenic liver cirrhosis & 1 & 5.9 & \\
\hline MELD score ${ }^{\mathrm{a}}$ & & & $16 \pm 7$ \\
\hline Cold ischemic time (minutes) ${ }^{\mathrm{a}}$ & & & $341 \pm 137$ \\
\hline Time from transplant surgery to study enrolment (weeks) & & & $122 \pm 82$ \\
\hline Weight $(\mathrm{kg})$ of patient at time of enrolment & & & $69.4 \pm 10.9$ \\
\hline Mean tacrolimus daily dose (mg) & & & $3.2 \pm 1.4$ \\
\hline Weight-adjusted tacrolimus dose (mg/kg/day) & & & $0.0464 \pm 0.0206$ \\
\hline \multicolumn{4}{|l|}{ Immunosuppressants at time of enrolment } \\
\hline Tacrolimus alone & 15 & 88.2 & \\
\hline Tacrolimus + Mycophenolatemofetil & 2 & 11.8 & \\
\hline Bilirubin (umol/L) & & & $16.9 \pm 2.4$ \\
\hline Albumin $(\mathrm{g} / \mathrm{L})$ & & & $41.8 \pm 2.5$ \\
\hline Alanine aminotransferase, ALT (U/L) & & & $33.8 \pm 35.3$ \\
\hline Aspartate aminotransferase, AST (U/L) & & & $29.7 \pm 12.4$ \\
\hline Alkaline phosphatase, ALP (U/L) & & & $69.6 \pm 20.3$ \\
\hline Serum creatinine, (umol/L) & & & $110.1 \pm 27.7$ \\
\hline Estimated creatinine clearance $(\mathrm{mL} / \mathrm{min})$ & & & $69.7 \pm 20.8$ \\
\hline Hematocrit (\%) & & & $41.0 \pm 4.1$ \\
\hline
\end{tabular}

${ }^{\mathrm{a}}$ for transplant recipients whose donor liver tissue samples were available $(\mathrm{n}=13)$. 
transplant $(\mathrm{n}=14,82.4 \%)$. Fifteen patients $(88.2 \%)$ were on tacrolimusmonotherapy. Mean $( \pm \mathrm{SD})$ tacrolimus daily dose and weight-adjusted dose was $3.2( \pm 1.4) \mathrm{mg}$ and $0.0464( \pm 0.0206) \mathrm{mg} / \mathrm{kg} / \mathrm{day}$, respectively. All patients with hepatitis B pre-transplant $(\mathrm{n}=12,70.6 \%)$ were on oral nucleoside analogue (lamivudine and/or adefovir or entecavir) and intramuscular hepatitis B immunoglobulin. At the time of the study, $8(47.0 \%)$ and $6(35.3 \%)$ patients were on antihypertensive and diabetic medications, respectively. Amlodipine and atenolol were the two most commonly prescribed antihypertensive agents. Out of 6 diabetic patients, 4 were on insulin and others were on glipizide and metformin. Other medications prescribed included co-trimoxazole $(\mathrm{n}=16,94.1 \%)$, omeprazole $(\mathrm{n}$ $=12,70.6 \%)$, calcium with vitamin $\mathrm{D}(\mathrm{n}=10,58.8 \%)$, aspirin $(\mathrm{n}=7,41.2 \%)$, statin $(\mathrm{n}=2,11.8 \%)$, clopidogrel $(\mathrm{n}=1,5.9 \%)$ and risedronate $(\mathrm{n}=1,5.9 \%)$.

\subsection{Pharmacokinetics of Tacrolimus}

The mean pharmacokinetic parameters of tacrolimus are presented in Table 2.

\subsection{Genotypes and C0/D Ratio}

DNA from a total of 13 donor liver tissue samples and 17 recipient blood samples were genotyped. Genotypes of recipients and donors; and respective $\mathrm{C} 0 / \mathrm{D}$ ratios are shown in Tables 3 and $\mathbf{4}$. No CYP3A4 polymorphism was detected in our study.

\subsection{Genotypes and Apparent Oral Clearance}

Apparent oral clearance $(\mathrm{CL} / \mathrm{F})$ at more than 3 months post transplant, according to respective donor and recipient genotypes, is presented in Table 5.

\subsection{Donor Genotypes}

In donor livers, the CYP3A5 genotype frequencies were $30.8 \%(\mathrm{n}=4)$ for genotype ${ }^{*}{ }^{*} 1,7.7 \%(\mathrm{n}=1)$ for genotype ${ }^{*}{ }^{*} 3$ and $61.5 \%(n=8)$ for genotype ${ }^{*}{ }^{*} 3$ (Table 3 ). At Week 1 post liver transplant, patients with liver grafts from CYP3A5 expressors $\left({ }^{*} 1^{*} 1\right.$ and $\left.{ }^{*} 1^{*} 3\right)$ demonstrated higher mean weight-adjusted dose than non-expressors $\left({ }^{*} 3^{*} 3\right)(0.12$ versus $0.08 \mathrm{mg} / \mathrm{kg}, \mathrm{P}=0.045)$ (Figure 1(a)). Not surprisingly, those with liver grafts from CYP3A5 expressors achieved lower mean $\mathrm{C} 0 / \mathrm{D}$ ratio than those with grafts from non-expressors (64.48 versus 129.21 $(\mathrm{mcg} / \mathrm{L}) /(\mathrm{mg} / \mathrm{kg} /$ day), $\mathrm{P}=0.040)$ (Figure 2(a)).

Donor liver CYP3A5 genotypes affected tacrolimus $\mathrm{C} 0 / \mathrm{D}$ ratio only at Week 1 , but not after 3 months post transplant (Table 3, Figure 2). In other words, the tacrolimus dose required to achieve target concentration at Week 1 post transplant was higher in patients with liver grafts from CYP3A5 expressors. All ABCB1 variants of donors did not significantly affect the $\mathrm{C} 0 / \mathrm{D}$ ratio (Table 3).

\subsection{Recipient Genotypes}

C0/D ratio did not show statistically significant difference according to any recipients' own gene polymerphisms (Table 4). At more than 3 months post transplant, mean weight-adjusted dose among recipient CYP3A5 expressors and non-expressors were not different $(0.05$ versus $0.04 \mathrm{mg} / \mathrm{kg}, \mathrm{P}=0.609$ ) (Figure 1(b)). Similar to donor, recipients who were CYP3A5 expressors have lower mean C0/D ratios than non-expressors (at Week 1: 86.58 versus $113.49(\mathrm{mcg} / \mathrm{L}) /(\mathrm{mg} / \mathrm{kg} /$ day $), P=1.000$; at more than 3 months post transplant: 135.31 versus $148.81(\mathrm{mcg} / \mathrm{L}) /(\mathrm{mg} / \mathrm{kg} /$ day), $\mathrm{P}=0.630)$ (Figure 3).

At more than 3 months post transplant, $\mathrm{CL} / \mathrm{F}$ was generally lower for recipient wild type of ABCB1 C3435T, G2677T/A and C1236T than for polymorphic variants (Table 5). CL/F wasstatistically significantly different among the recipient genotypes of $\mathrm{ABCB} 1 \mathrm{C} 3435 \mathrm{~T}(\mathrm{P}=$ 0.044 ), with the TT variant having higher $\mathrm{CL} / \mathrm{F}$ than the $\mathrm{CC}$ variant (wild type) $(17.7$ versus $7.9 \mathrm{~L} / \mathrm{h}, \mathrm{P}=0.033)$ (Table 5).

\subsection{Donor/Recipient Genotype Combinations}

After excluding the single patient of expressor/expressor, mean $\mathrm{C} 0 / \mathrm{D}$ ratio was statistically different at Week 1 (P

Table 2. Steady-state pharmacokinetic parameters of tacrolimus in study transplant recipients $(\mathrm{n}=\mathbf{1 7})$.

\begin{tabular}{cc}
\hline & Mean $\pm \mathrm{SD}$ \\
\hline Area under the concentration-time curve at steady state within a 12-h dosing interval, $\mathrm{AUC}_{\mathrm{ss}, 0-12}(\mathrm{mcg} . \mathrm{h} / \mathrm{L})$ & $187.0 \pm 94.2$ \\
Trough concentration, $\mathrm{C} 0(\mathrm{mcg} / \mathrm{L})$ & $5.8 \pm 2.2$ \\
Maximum concentration, Cmax $(\mathrm{mcg} / \mathrm{L})$ & $12.4 \pm 4.7$ \\
Time to reach maximum concentration, Tmax (h) & $1.7 \pm 0.7$ \\
Elimination rate constant,Ke $\left(\mathrm{h}^{-1}\right)$ & $0.085 \pm 0.030$ \\
Half-life, $\mathrm{t}_{\mathrm{l} / 2}(\mathrm{~h})$ & $9.4 \pm 4.8$ \\
Apparent oral clearance, $\mathrm{CL} / \mathrm{F}(\mathrm{L} / \mathrm{h})$ & $10.1 \pm 5.6$ \\
Apparent volume of distribution, $\mathrm{V} / \mathrm{F}(\mathrm{L})$ & $119.8 \pm 48.4$ \\
\hline
\end{tabular}


Table 3. Donor genotypes and C0/D ratio at Week 1 and at more than 3 months from Transplant $(n=13)$.

\begin{tabular}{|c|c|c|c|c|c|c|c|c|}
\hline Gene & Position & Genotype & Donor & $\begin{array}{c}\mathrm{C} 0 / \mathrm{D} \text { ratio at Week } 1 \\
(\mathrm{mcg} / \mathrm{L}) /(\mathrm{mg} / \mathrm{kg} / \mathrm{day}) \\
(\mathrm{mean} \pm \mathrm{SD})\end{array}$ & $P$ value & $\begin{array}{l}\mathrm{C} 0 / \mathrm{D} \text { ratio at more } \\
\text { than } 3 \text { months from } \\
\text { transplant } \\
(\mathrm{mcg} / \mathrm{L}) /(\mathrm{mg} / \mathrm{kg} / \mathrm{day}) \\
(\mathrm{mean} \pm \mathrm{SD})\end{array}$ & $P$ value & \\
\hline & & & $\mathrm{n}$ & $\%$ & & & & \\
\hline \multirow[t]{9}{*}{$\mathrm{ABCB} 1$} & G2677T/A & GG & 4 & 30.8 & $55.9 \pm 17.7$ & 0.056 & $110.4 \pm 34.3$ & 0.164 \\
\hline & & $\mathrm{G}(\mathrm{T} / \mathrm{A})$ & 5 & 38.5 & $150.9 \pm 95.8$ & & $162.5 \pm 77.1$ & \\
\hline & & $\mathrm{TT}$ & 4 & 30.8 & $94.5 \pm 25.1$ & & $134.0 \pm 1.94$ & \\
\hline & $\mathrm{C} 3435 \mathrm{~T}$ & $\mathrm{CC}$ & 5 & 38.5 & $81.9 \pm 60.0$ & 0.321 & $139.0 \pm 70.5$ & 0.647 \\
\hline & & $\mathrm{CT}$ & 4 & 30.8 & $142.2 \pm 108.3$ & & $139.8 \pm 66.9$ & \\
\hline & & $\mathrm{TT}$ & 4 & 30.8 & $94.5 \pm 25.1$ & & $134.0 \pm 1.9$ & \\
\hline & $\mathrm{C} 1236 \mathrm{~T}$ & $\mathrm{CC}$ & 0 & 0 & & 0.643 & & 0.557 \\
\hline & & $\mathrm{CT}$ & 4 & 30.8 & $146.9 \pm 120.2$ & & $147.7 \pm 90.3$ & \\
\hline & & $\mathrm{TT}$ & 9 & 69.2 & $85.4 \pm 28.1$ & & $133.2 \pm 32.4$ & \\
\hline \multirow[t]{3}{*}{ CYP $3 \mathrm{~A} 5^{*} 3$} & A6986G & AA $\left({ }^{*} 1^{*} 1\right)$ & 4 & 30.8 & $68.7 \pm 26.2$ & 0.100 & $124 \pm 47.9$ & 0.595 \\
\hline & & $\mathrm{AG}\left({ }^{*} 1^{*} 3\right)$ & 1 & 7.7 & 47.6 & & 120.6 & \\
\hline & & GG $\left({ }^{*} 3^{*} 3\right)$ & 8 & 61.5 & $129.2 \pm 80.0$ & & $146.6 \pm 59.6$ & \\
\hline CYP $3 A^{*} 3$ & & TT & 13 & 100 & $104.3 \pm 70.8$ & & $142.5 \pm 62.4$ & \\
\hline
\end{tabular}

Table 4. Recipient genotypes and C0/D ratio at Week 1 and at more than 3 months from transplant $(n=17)$.

\begin{tabular}{|c|c|c|c|c|c|c|c|c|}
\hline Gene & Position & Genotype & Recipient & $\begin{array}{c}\mathrm{C} 0 / \mathrm{D} \text { ratio at } \\
\text { Week } 1(\mathrm{mcg} / \mathrm{L}) / \\
(\mathrm{mg} / \mathrm{kg} / \mathrm{day}) \\
(\mathrm{mean} \pm \mathrm{SD})\end{array}$ & $P$ value & $\begin{array}{c}\mathrm{C} 0 / \mathrm{D} \text { ratio at more } \\
\text { than } 3 \text { months from } \\
\text { transplant }(\mathrm{mcg} / \mathrm{L}) / \\
(\mathrm{mg} / \mathrm{kg} / \mathrm{day}) \\
(\text { mean } \pm \mathrm{SD})\end{array}$ & $P$ value & \\
\hline & & & $\mathrm{n}$ & $\%$ & & & & \\
\hline \multirow[t]{9}{*}{$\mathrm{ABCB} 1$} & G2677T/A & GG & 4 & 23.5 & $91.8 \pm 30.3$ & 0.889 & $149.8 \pm 25.6$ & 0.055 \\
\hline & & $\mathrm{G}(\mathrm{T} / \mathrm{A})$ & 7 & 47.1 & $129.6 \pm 112.8$ & & $164.5 \pm 79.6$ & \\
\hline & & $\mathrm{TT} / \mathrm{AT}$ & 6 & 29.4 & $85.0 \pm 41.5$ & & $101.4 \pm 29.4$ & \\
\hline & C3435T & $\mathrm{CC}$ & 9 & 52.9 & $98.5 \pm 46.1$ & 0.763 & $168.9 \pm 70.1$ & 0.061 \\
\hline & & $\mathrm{CT}$ & 5 & 29.4 & $119.7 \pm 106.7$ & & $132.2 \pm 33.0$ & \\
\hline & & $\mathrm{TT}$ & 3 & 17.6 & $75.4 \pm 49.4$ & & $80.1 \pm 5.7$ & \\
\hline & $\mathrm{C} 1236 \mathrm{~T}$ & $\mathrm{CC}$ & 4 & 23.5 & $129.9 \pm 48.4$ & 0.184 & $191.4 \pm 49.0$ & 0.055 \\
\hline & & $\mathrm{CT}$ & 3 & 17.6 & $51.4 \pm 24.3$ & & $85.5 \pm 46.6$ & \\
\hline & & TT & 10 & 58.8 & $107.8 \pm 79.7$ & & $140.0 \pm 59.0$ & \\
\hline \multirow[t]{3}{*}{ CYP $3 \mathrm{~A}^{*} 3$} & A6986G & $\mathrm{AA}\left({ }^{*} 1^{*} 1\right)$ & 2 & 11.8 & $64.2 \pm 14.0$ & 0.756 & $86.2 \pm 65.9$ & 0.406 \\
\hline & & $\operatorname{AG}\left({ }^{*} 1^{*} 3\right)$ & 6 & 35.3 & $95.5 \pm 41.0$ & & $167.2 \pm 69.1$ & \\
\hline & & $\mathrm{GG}\left({ }^{*} 3^{*} 3\right)$ & 9 & 52.9 & $113.5 \pm 89.4$ & & $148.8 \pm 55.2$ & \\
\hline CYP3A4*3 & & TT & 17 & 100 & $104.3 \pm 70.8$ & & $142.5 \pm 62.4$ & \\
\hline
\end{tabular}

$=0.049)$ but not for after 3 months from transplant $(\mathrm{P}=$ 0.246 ) according to combination of CYP3A5 genotypes in both donor and recipient (Table 6). The difference in $\mathrm{C} 0 / \mathrm{D}$ ratio was only statistically significant between ex- pressor/non-expressor and non-expressor/non-expressor $(\mathrm{P}=0.043)$. Nonetheless, combinations with expressor (expressor/expressor, expressor/non-expressor and nonexpressor/expressor) appeared to have lower $\mathrm{C} 0 / \mathrm{D}$ ratio 


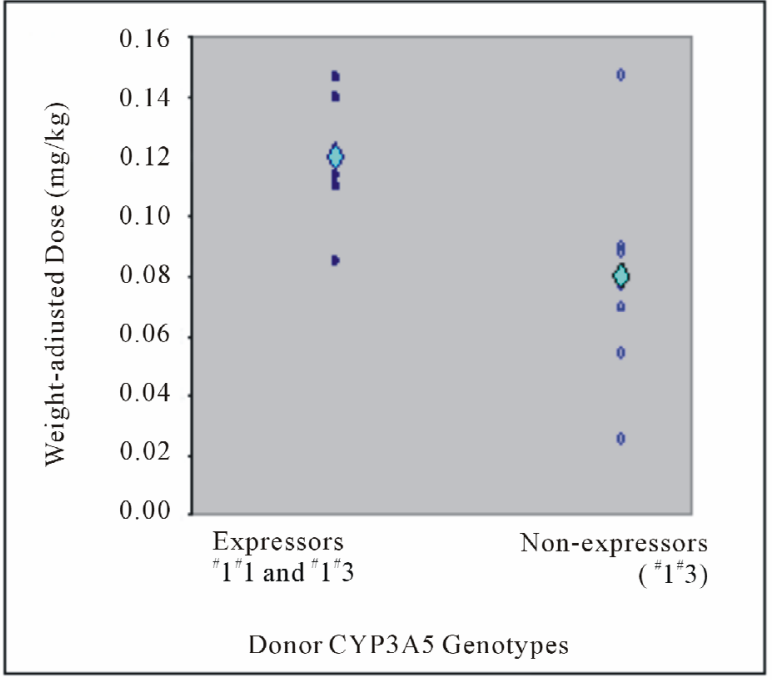

(a)

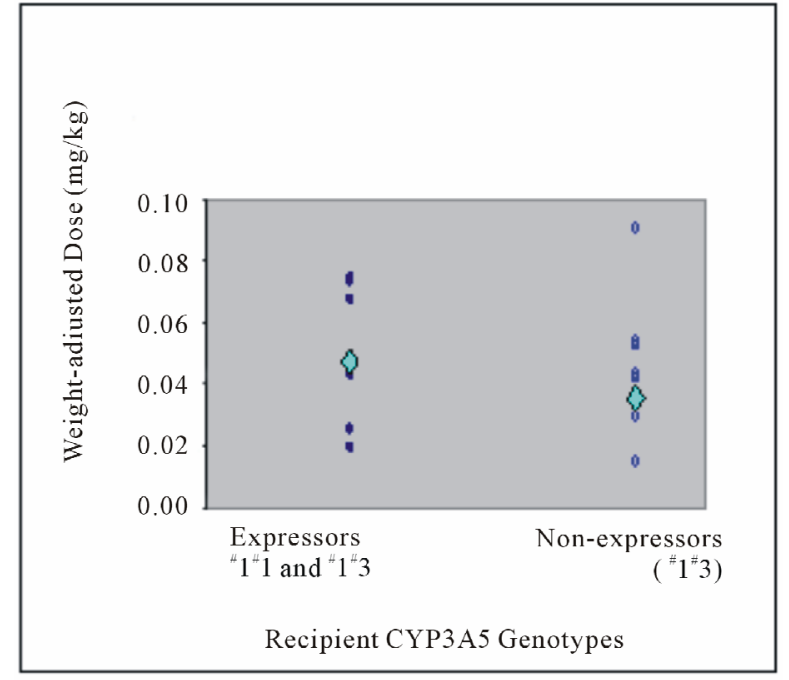

(b)

Figure 1. Weight-adjusted doses at (a) Week 1 post liver transplant for liver transplant recipients with respective donor CYP3A5 genotypes and (b) more than 3 months from transplant for liver transplant recipients with respective recipient CYP3A5 genotypes. The mean weight-adjusted doses are depicted by the light blue diamond.

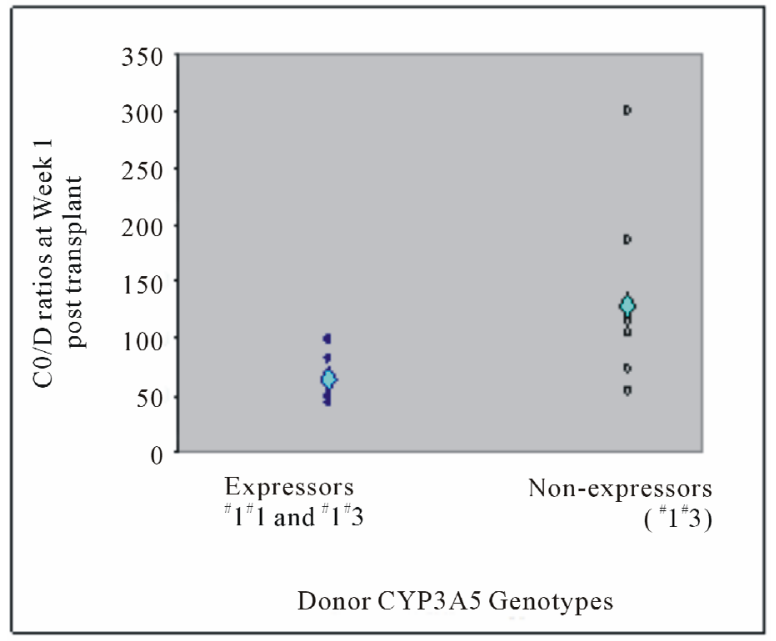

(a)

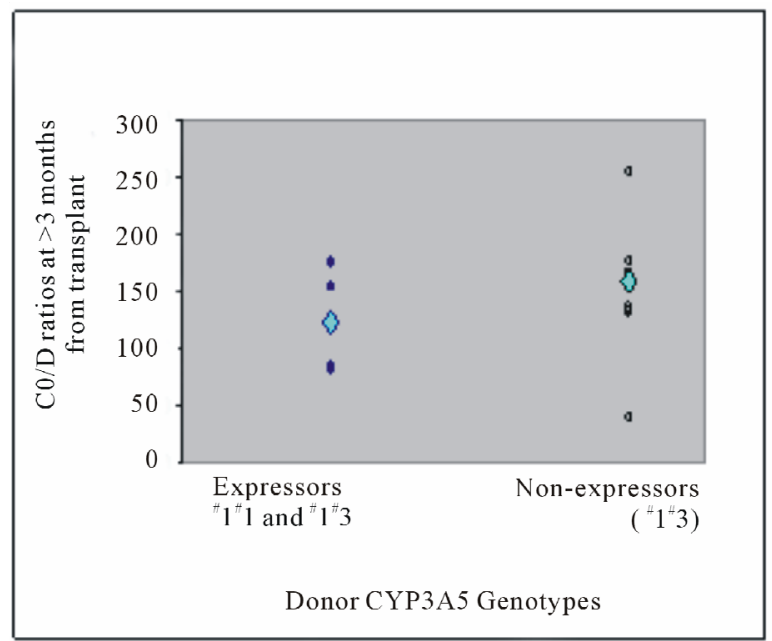

(b)

Figure 2. Tacrolimus C0/D ratios (mcg/L)/(mg/kg/day) at (a) Week 1 post liver transplant and (b) more than 3 months from transplant of liver transplant recipients with respective donor CYP3A5 genotypes. The mean C0/D ratios are depicted by the light blue diamond.

than non-expressor/non-expressor combination.

\section{Discussion}

In liver transplantation, the native liver is being replaced with a donor liver that may likely share different genotypes from the recipient. As most of the metabolising enzymes are in the liver, the influence of donor liver genotypes should be considered. However, one of the most important predictors of early tacrolimus metabolism (at Week 1) is immediate graft function. This, in turn, is influenced by multiple donor and recipient factors which may be independent of genotype (such as ischemic reperfusion injury and recipient condition in the perioperative period (e.g. MELD score)). Hence, making it difficult to predict and less likely for tacrolimus concentration at Week 1 post transplant surgery to be at steady state.

The time point at which the C0/D ratio was influenced by genotypes was not clearly stated in many studies. In our study, recipient genotype potentially has greater influence with time elapsed post-transplant, however the specific time-point at which it affects metabolism is beyond the scope of this study.

At Week 1, individuals with donor liver CYP3A5*1 


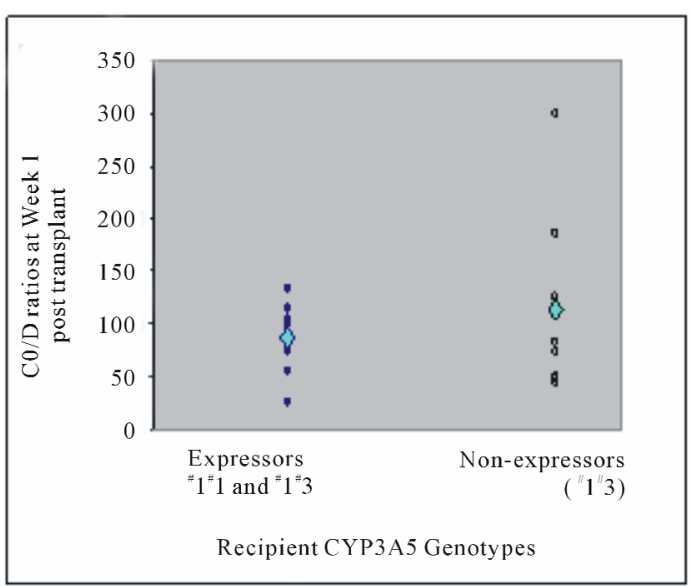

(a)

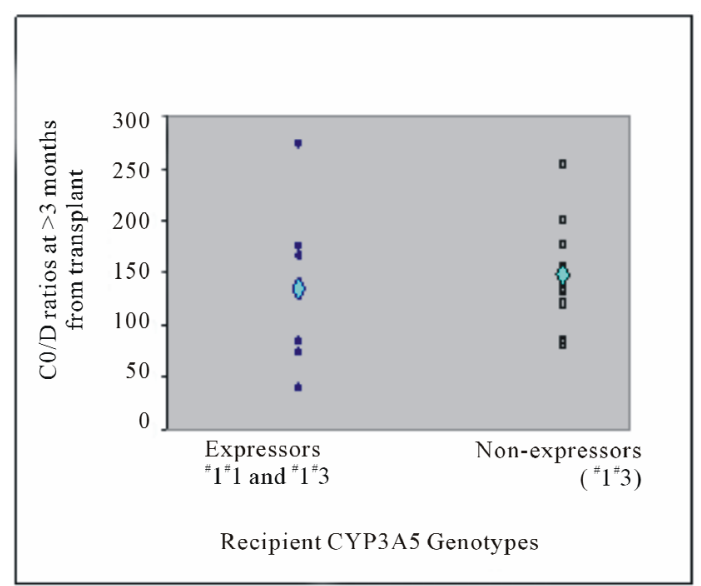

(b)

Figure 3. Tacrolimus C0/D ratios (mcg/L)/(mg/kg/day) at (a) Week 1 post liver transplant and (b) more than 3 months from transplant of liver transplant recipients with respective CYP3A5 genotypes. The mean C0/D ratios are depicted by the light blue diamond.

Table 5. Apparent Oral Clearance at more than 3 Months from Transplant $(n=17)$.

\begin{tabular}{|c|c|c|c|c|c|c|c|c|c|c|}
\hline & & & & & Donor & & & & Recipient & \\
\hline Gene & Position & Genotype & $\mathrm{n}$ & $\%$ & $\begin{array}{c}\mathrm{CL} / \mathrm{F}(\mathrm{L} / \mathrm{h}) \\
(\text { mean } \pm \mathrm{SD})\end{array}$ & $P$ value & $\mathrm{n}$ & $\%$ & $\begin{array}{c}\mathrm{CL} / \mathrm{F}(\mathrm{L} / \mathrm{h}) \\
(\text { mean } \pm \mathrm{SD})\end{array}$ & $P$ value \\
\hline \multirow{9}{*}{$\mathrm{ABCB} 1$} & G2677T/A & GG & 4 & 30.8 & $14.2 \pm 5.9$ & \multirow{3}{*}{0.062} & 4 & 23.5 & $6.3 \pm 2.8$ & \multirow{3}{*}{0.155} \\
\hline & & $\mathrm{G}(\mathrm{T} / \mathrm{A})$ & 5 & 38.5 & $8.4 \pm 7.0$ & & 7 & 47.1 & $9.6 \pm 6.1$ & \\
\hline & & $\mathrm{TT} / \mathrm{AT}$ & 4 & 30.8 & $8.0 \pm 1.7$ & & 6 & 29.4 & $13.3 \pm 5.5$ & \\
\hline & $\mathrm{C} 3435 \mathrm{~T}$ & $\mathrm{CC}$ & 5 & 38.5 & $12.3 \pm 6.6$ & \multirow{3}{*}{0.337} & 9 & 52.9 & $7.9 \pm 5.2$ & \multirow{3}{*}{0.044} \\
\hline & & $\mathrm{CT}$ & 4 & 30.8 & $9.3 \pm 7.7$ & & 5 & 29.4 & $9.7 \pm 3.2$ & \\
\hline & & $\mathrm{TT}$ & 4 & 30.8 & $8.0 \pm 1.7$ & & 3 & 17.6 & $17.7 \pm 3.9$ & \\
\hline & $\mathrm{C} 1236 \mathrm{~T}$ & $\mathrm{CC}$ & 0 & 0 & & \multirow{3}{*}{0.877} & 4 & 23.5 & $5.5 \pm 0.7$ & \multirow{3}{*}{0.068} \\
\hline & & $\mathrm{CT}$ & 4 & 30.8 & $10.5 \pm 7.0$ & & 3 & 17.6 & $14.7 \pm 6.2$ & \\
\hline & & $\mathrm{TT}$ & 9 & 69.2 & $9.9 \pm 5.6$ & & 10 & 58.8 & $10.6 \pm 5.7$ & \\
\hline \multirow{3}{*}{ CYP $3 \mathrm{~A}^{*} 3$} & A6986G & $\mathrm{AA}\left({ }^{*} 1^{*} 1\right)$ & 4 & 30.8 & $13.3 \pm 6.9$ & \multirow{3}{*}{0.126} & 2 & 11.8 & $14.4 \pm 8.8$ & \multirow{3}{*}{0.182} \\
\hline & & $\mathrm{AG}\left({ }^{*} 1^{*} 3\right)$ & 1 & 7.7 & 10 & & 6 & 35.3 & $8.6 \pm 5.6$ & \\
\hline & & $\mathrm{GG}\left({ }^{*} 3^{*} 3\right)$ & 8 & 61.5 & $8.5 \pm 5.4$ & & 9 & 52.9 & $10.2 \pm 5.4$ & \\
\hline
\end{tabular}

Table 6. Tacrolimus C0/D ratio in groups of donor/recipient CYP3A5 genotype combination at Week 1 and more than 3 months from transplant $(n=13)$.

\begin{tabular}{|c|c|c|c|c|c|}
\hline $\begin{array}{l}\text { Combination of donor/ recipient } \\
\text { CYP3A5 genotypes }\end{array}$ & $\mathrm{n}$ & $\begin{array}{l}\mathrm{C} 0 / \mathrm{D} \text { ratio at Week } 1 \\
(\mathrm{mcg} / \mathrm{L}) /(\mathrm{mg} / \mathrm{kg} / \text { day }) \\
(\text { mean } \pm \mathrm{SD})\end{array}$ & $P$ value & $\begin{array}{l}\mathrm{C} 0 / \mathrm{D} \text { ratio at more than } 3 \\
\text { months from transplant } \\
(\mathrm{mcg} / \mathrm{L}) /(\mathrm{mg} / \mathrm{kg} / \text { day }) \\
(\text { mean } \pm \mathrm{SD})\end{array}$ & $P$ value \\
\hline Expressor/Expressor & 1 & 98.8 & \multirow{4}{*}{0.114} & 175.1 & \multirow{4}{*}{0.237} \\
\hline Expressor/Non-expressor & 4 & $55.9 \pm 17.7$ & & $110.4 \pm 34.3$ & \\
\hline Non-expressor/Expressor & 4 & $87.3 \pm 28.2$ & & $119.0 \pm 55.1$ & \\
\hline Non-expressor/Non-expressor & 4 & $171.1 \pm 97.4$ & & $174.3 \pm 56.7$ & \\
\hline
\end{tabular}

Expressor: ${ }^{*} 1{ }^{*} 1$ and ${ }^{*} 1 * 3$, Non-expressor: ${ }^{*} 3{ }^{*} 3$. 
expressors (both ${ }^{*} 1{ }^{*} 1$ and ${ }^{*} 1{ }^{*} 3$ ) demonstrated lower C0/D ratio than non-expressors $\left({ }^{*} 3^{*} 3\right)$. This could be due to the higher rates of metabolism in CYP3A5 expressors and thus, the dose required to achieve target concentration in patients with donor genotype CYP3A5 expressors was higher than non-expressors. Our finding was consistent with results from other studies [5,6,9]. However, similar impact on $\mathrm{C} 0 / \mathrm{D}$ ratio was not demonstrated in patients with donor $\mathrm{ABCB} 1$ variant alleles.

Donor CYP3A and ABCB1 genotypes did not seem to affect $\mathrm{C} 0 / \mathrm{D}$ ratio at more than 3 months post transplant. This finding was not unexpected. Donor lymphocyte chimerism post organ transplant may change with time elapsed transplant [22]. Chimerism, the coexisting of donor and recipient immune system [23], could have complicated the investigation in post liver transplant recipients.

In contrast, none of the recipient genetic variants showed significant influence on tacrolimus $\mathrm{C} 0 / \mathrm{D}$ ratio at 1 week immediate post transplant, as well as after 3 months post transplant. Nevertheless, tacrolimus $\mathrm{CL} / \mathrm{F}$ was significantly affected by recipient ABCB1 C3435T at more than 3 months post transplant. Variant alleles of ABCB1 C1236T, C3435T and G2677A/T may potentially affect tacrolimus clearance rate.

Interaction between donor and recipient CYP3A5 genotype was investigated. Again, expressor combinations (i.e. expressor/expressor, expressor/non-expressor or nonexpressor/expressor) could have higher metabolism rate at Week 1 post transplant and thus lower C0/D ratio. However, pairwise analysis of linkage disequilibrium of variant alleles, i.e. the interaction between two or more SNPs (donors' only and recipients' only, and combination of donors and recipients) could not be carried out due to our small sample size. This is a limitation of our study, as such an analysis would have been more optimal in shedding light on the influence of genotypes on the metabolism of tacrolimus.

In this era, genotyping turnaround time could be as fast as 24 hours. Given the high prevalence of CYP3A5 expressors $(38 \%$ among donors and $47 \%$ among recipients in this study, $51 \%$ in study by Loh PT, et al [8], genotyping could be cost-effective instead of merely adjusting tacrolimus dose according to daily therapeutic drug monitoring. Therapeutic tacrolimus concentration could potentially be achieved in a shorter period. Prevalence of important single nucleotide polymorphisms that influence CYP3A enzyme and ABCB1 activity in this cohort study could potentially assist in better understanding of the inter-individual variability of tacrolimus dose requirement. Further studies are required to determine dosing recommendation according to CYP3A5 and $\mathrm{ABCB} 1$ genotypes. The word "data" is plural, not singular.

\section{Conclusion}

Donor liver CYP3A polymorphism could potentially affect tacrolimus C0/D ratio as early as the first week post liver transplant. Genotyping of liver donors may be useful in dosing to achieve the optimal drug level during this critical period. Recipient genotypes appeared to influence steady-state tacrolimus pharmacokinetics, however, the explicit time point at which impact becomes significant requires further investigation. Further prospective studies with a larger population are needed to illustrate mechanism and impact of gene polymorphisms on metabolism of tacrolimus.

\section{Acknowledgements}

One of us, Mei-Ling Yee thanks Dr. Kiat-Hon Lim, FRCP, Consultant, Department of Pathology, Dr. Yi Zhao, PhD, Research Scientist, Department of Clinical Research and Dr. Andrea Lay Hoon Kwa, PharmD, Research Scientist, Department of Pharmacy, Singapore General Hospital, Singapore.

\section{REFERENCES}

[1] The US Multicenter FK506 Liver Study Group, "A Comparison of Tacrolimus (FK 506) and Cyclosporine for Immunosuppression in Liver Transplantation," The New England Journal of Medicine, Vol. 331, No. 17, 1994, pp. 1110-1115.

[2] European FK506 Multicenter Liver Study Group, "Randomised Trial Comparing Tacrolimus (FK506) and Cycloporin in Prevention of Liver Allograft Rejection," Lancet, Vol. 344, No. 8920, 1994, pp. 423-428. doi:10.1016/S0140-6736(94)91766-3

[3] J. J. Fung, M. Eliasziw, S. Todo et al., "The Pittsburgh Randomized Trial of Tacrolimus Compared to Cyclosporine for Hepatic Transplantation," Journal of the American College of Surgeons, Vol. 183, No. 2, 1996, pp. 117-125.

[4] D. Li, W. Lu, J.-Y. Zhu, et al., "Population Pharmacokinetic of Tacrolimus and CYP3A5, MDR1 and IL-10 Polymorphisms in Adult Liver Transplant Patients," Journal of Clinical Pharmacy and Therapeutics, Vol. 32, No. 5, 2007, pp. 505-515. doi:10.1111/j.1365-2710.2007.00850.x

[5] M. Goto, S. Masuda, T. Kiuchi, et al., "CYP3A5"1-Carrying Graft Liver Reduces the Concentration/Oral Dose Ratio of Tacrolimus in Recipients of Living-Donor Liver Transplantation," Pharmacogenetics, Vol. 14, No. 7, 2004, pp. 471-478. doi:10.1097/01.fpc.0000114747.08559.49

[6] S. F. Yu, L. H. Wu, J. Jin, et al., "Influence of CYP3A5 Gene Polymorphisms of Donor Rather Than Recipient to Tacrolimus Individual Dose Requirement in Liver Transplantation," Transplantation, Vol. 81, No. 1, 2006, pp. 46-51. doi:10.1097/01.tp.0000188118.34633.bf

[7] E. Laure, C. Arnaud, V. K. Valerie, et al., "1199G > A and $2677 \mathrm{G}>\mathrm{T} / \mathrm{A}$ Polymorphisms of ABCB1 Independ- 
ently Affect Tacrolimus Concentration in Hepatic Tissue after Liver Transplantation," Pharmacogenetics and Genomics, Vol. 17, No. 10, 2007, pp. 873-883.

[8] P. T. Loh, H. X. Lou, Y. Zhao, et al., "Significant Impact of Gene Polymorphisms on Tacrolimus But Not Cyclosporine Dosing in Asian Renal Transplant Recipients," Transplantation Proceedings, Vol. 40, No. 5, 2008, pp. 1690-1695. doi:10.1016/j.transproceed.2008.04.010

[9] K. R. Jun, W. C. Lee, M. S. Jang, et al., "Tacrolimus Concentrations in Relation to CYP3A and ABCB1 Polymorphisms among Solid Organ Transplant Recipient in Korea," Transplantation, Vol. 87, No. 8, 2009, pp. 12251231. doi:10.1097/TP.0b013e31819f117e

[10] T. Eric, A. Dany, K. Barry, et al., "Impact of Cytochrome P450 3A5 Genetic Polymorpisms on Tacrolimus Doses and Concentration-to-Dose Ratio in Renal Transplant Recipients," Transplantation, Vol. 76, No. 8, 2003, pp. 1233-1235.

[11] H. Zahir, A. J. McLachlan, A. Nelson, et al., "Population Pharmacokinetic Estimation of Tacrolimus Apparent Clearance in Adult Liver Transplant Recipients," Therapeutic Drug Monitoring, Vol. 27, No. 4, 2005, pp. 422-430. doi:10.1097/01.ftd.0000170029.36573.a0

[12] V. Mardigyan, J. Tchervenkov, P. Metrakos, et al., "Best Single Time Points as Surrogates to the Tacrolimus and Mycophenolic Acid Area under the Curve in Adult Liver Transplant Patients Beyond 12 Months of Transplantation," Clinical Therapeutics, Vol. 27, No. 4, 2005, pp. 463-468. doi:10.1016/j.clinthera.2005.04.004

[13] M. Cantarovich, J. Fridell, J. Barkun, et al., "Optimal Time Points for the Prediction of the Area-Under-TheCurve in Liver Transplant Patients Receiving Tacrolimus," Transplantation Proceedings, Vol. 30, No. 4, 1998, pp. 1460-1461. doi:10.1016/S0041-1345(98)00315-7

[14] A. Jain, R. Venkataramanan, R. Sharma, et al., "Pharmacokinetics of Tacrolimus in Living Donor Liver Transplant and Deceased Donor Liver Transplant Recipients," Transplantation, Vol. 85, No. 4, 2008, pp. 554-560. doi:10.1097/TP.0b013e3181642c95
[15] IMx “Tacrolimus II Package Insert," Abbott Laboratories, Diagnostic Division, Revised September 2007.

[16] D. I. Min, H. Y. Chen, A. Fabrega, et al., "Circadian Variation of Tacrolimus Disposition in Liver Allograft Recipients," Transplantation, Vol. 62, No. 8, 1996, pp. 1190-1192. doi:10.1097/00007890-199610270-00031

[17] R. Venkataramanan, A. Swaminathan, T. Prasad, et al., "Clinical Pharmacokinetics of Tacrolimus," Clinical Pharmacokinetics, Vol. 29, No. 6, 1995, pp. 404-30. doi:10.2165/00003088-199529060-00003

[18] G. Plosker and R. Foster, "Tacrolimus-A Further Update of its Pharmacology and Therapeutic Use in the Management of Organ Transplantation," Drugs, Vol. 59, No. 2, 2000, pp. 323-389. doi:10.2165/00003495-200059020-00021

[19] W. J. Sam, L. S. Tham, M. J. Holmes, et al., "Population Pharmacokinetics of Tacrolimus in Whole Blood and Plasma in Asian Liver Transplant Patients," Clinical Pharmacokinetics, Vol. 45, No. 1, 2006, pp. 59-75. doi:10.2165/00003088-200645010-00004

[20] J. Y. Lee, H. J. Hahn, I. J. Son, et al., "Factors Affecting the Apparent Clearance of Tacrolimus in Korean Adult Liver Transplant Recipients," Pharmacotherapy, Vol. 26, No. 8, 2006, pp. 1067-1077.

[21] H. Zahir, G. McCaughan, M. Gleeson, et al., "Factors Affecting Variability in Distribution of Tacrolimus in Liver Transplant Recipients," British Journal of Clinical Pharmacology, Vol. 57, No. 3, 2004, pp. 298-309.

[22] R. Domiati-Saad, G. B. Klintmalm, G. Netto, et al., "Acute Graft versus Host Disease after Liver Transplantation: Patterns of Lymphocyte Chimerism," American Journal of Transplantation, Vol. 5, No. 12, 2005, pp. 2968-2973. doi:10.1111/j.1600-6143.2005.01110.x

[23] T. E. Starzl, "Chimerism and Tolerance in Transplantation," Proceedings of the National Academy of Sciences, Vol. 101, Suppl. 2, 5 October 2004, pp. 14607-14614. 